Fayetteville State University

DigitalCommons@Fayetteville State University

Math and Computer Science Faculty Working

Papers

Math and Computer Science

2-14-2011

\title{
Lie Derivable Mappings on Prime Rings
}

Wu Jing

Fayetteville State University, wjing@uncfsu.edu

Fangyan Lu

Soochow University

Follow this and additional works at: https://digitalcommons.uncfsu.edu/macsc_wp

\section{Recommended Citation}

Jing, Wu and Lu, Fangyan, "Lie Derivable Mappings on Prime Rings" (2011). Math and Computer Science Faculty Working Papers. 7.

https://digitalcommons.uncfsu.edu/macsc_wp/7

This Article is brought to you for free and open access by the Math and Computer Science at DigitalCommons@Fayetteville State University. It has been accepted for inclusion in Math and Computer Science Faculty Working Papers by an authorized administrator of DigitalCommons@Fayetteville State University. For more information, please contact dballar5@uncfsu.edu. 


\title{
LIE DERIVABLE MAPPINGS ON PRIME RINGS
}

\author{
WU JING AND FANGYAN LU
}

\begin{abstract}
Let $R$ be a prime ring containing a nontrivial idempotent. Suppose that a mapping $\delta: R \rightarrow R$ satisfies

$$
\delta([a, b])=[\delta(a), b]+[a, \delta(b)]
$$

for all $a, b \in R$. Then there exists a $z_{a, b}$ (depending on $a$ and $b$ ) in its center $Z(R)$ such that

$$
\delta(a+b)=\delta(a)+\delta(b)+z_{a, b} .
$$

Moreover, if $R$ is 2-torsion free additionally, then $\delta$ is of the form $D+\tau$, where $D$ is a derivation of $R$ into its central closure $T$ and $\tau$ is a mapping of $R$ into its extended centroid $C$ such that $\tau(a+b)=\tau(a)+\tau(b)+z_{a, b}$ and $\tau([a, b])=0$ for all $a, b \in R$
\end{abstract}

\section{INTRODUCTION AND PRELIMINARIES}

The motivations of this paper are the additivity of mappings on rings and characterization of Lie derivations on prime rings.

In recent years, the additivity of mappings on rings has attracted the attentions of many researchers. These mappings include multiplicative maps, (Jordan) derivable mappings, Jordan (triple) mappings, Jordan elementary mappings, and so on (see [1], [4]-[8], and references therein). For example, in his pioneer paper [8], Martindale III obtained the following result.

Theorem 1.1. Let $R$ be a ring containing a family $\left\{e_{\alpha}: \alpha \in \Lambda\right\}$ of idempotents which satisfies

(1) $x R=\{0\}$ implies $x=0$.

(2) If $e_{\alpha} R x=\{0\}$ for each $\alpha \in \Lambda$, then $x=0$ (and hence $R x=\{0\}$ implies $x=0)$.

(3) For each $\alpha \in \lambda, e_{\alpha} x e_{\alpha} R\left(1-e_{\alpha}\right)=\{0\}$ implies $e_{\alpha} x e_{\alpha}=0$.

Then any multiplicative bijective map from $R$ onto an arbitrary ring $R^{\prime}$ is additive.

In this paper, we want to continue the study of additivity of mappings on rings by investigating Lie derivable mappings on prime rings. Recall that a mapping $\delta$ from a ring $R$ into itself is called a Lie derivable mapping if

$$
\delta([a, b])=[\delta(a), b]+[a, \delta(b)]
$$

holds true for all $a, b \in R$, where $[a, b]=a b-b a$ is the usual Lie product of $a$ and $b$. Instead of being additive, we shall show that any Lie derivable mapping on any

2000 Mathematics Subject Classification. 16W25, 16N60.

Key words and phrases. Lie derivable map; Lie derivation; idempotent; Peirce decomposition; prime ring; central closure; extended centroid.

The first author is supported by Fayetteville State University STEM Program Research Grant and Course Reassignment Program. The second author is supported by NNSFC. 
prime ring $R$ is nearly additive in the sense that for any $a, b \in R$ there is a $z_{a, b}$ (depending on $a$ and $b$ ) in the center of $R$ such that $\delta(a+b)=\delta(a)+\delta(b)+z_{a, b}$.

Recall that an additive mapping from ring $R$ into itself is called a Lie derivation if

$$
\delta([a, b])=[\delta(a), b]+[a, \delta(b)]
$$

holds true for all $a, b \in R$.

There have been several results on the characterizations of Lie derivations on rings. The first characterization on Lie derivations is due to Martindale III who proved the following result in 1964 .

Theorem 1.2. ([9]) Let $L$ be a Lie derivation of a primitive ring $R$ into itself, where $R$ contains a nontrivial idempotent and the characteristic of $R$ is not 2 then every Lie derivation $L$ of $R$ is of the form $L=D+T$, where $D$ is an ordinary derivation of $R$ into a primitive ring $\bar{R}$ containing $R$ and $T$ is an additive mapping of $R$ into the center of $\bar{R}$ that maps commutators into zero.

In 1993, Brešar generalized the above characterization of Lie derivations on primitive rings to those on prime rings. He obtained the following theorem.

Theorem 1.3. ([3]) Let $R$ be a prime ring of characteristic not 2. Let $d$ be a Lie derivation of $R$. If $R$ does not satisfy $S_{4}$, then $d$ is of the form $\delta+\tau$, where $\delta$ is a derivation of $R$ into its central closure and $\tau$ is an additive mapping of $R$ into its extended centroid sending commutators to zero.

As for characterizations on Lie derivable mappings on operator algebras, the following result is proved in [7].

Theorem 1.4. Let $X$ be a Banach space of dimension greater than 1 and $\delta$ be a Lie derivable mapping of $B(X)$ into itself. Then $\delta=D+\tau$, where $D$ is an additive derivation and $\tau$ is a map from $B(X)$ into $\mathbb{F} I$ vanishing at commutators.

In this paper, we shall also generalize the above result on Lie derivable mappings to prime rings by showing that every Lie derivable mapping on prime rings can be expressed as the sum of an ordinary derivation $D$ and a central mapping $\tau$ which vanishes at each commutator and is nearly additive in the sense that for any $a, b \in R$ there is a $z_{a, b}$ (depending on $a$ and $b$ ) in its center such that $\tau(a+b)=$ $\tau(a)+\tau(b)+z_{a, b}$.

Before proceeding, we list some notations and results which will be used to prove our results. Let $R$ be an arbitrary ring with a nontrivial idempotent $e$. We write $e_{1}=e$ and $e_{2}=1-e_{1}$. Note that $R$ need not have identity element. Put $e_{i} R e_{j}=R_{i j}$ for any $i, j=1,2$. Then we have the Peirce decomposition of $R$ as $R=R_{11} \oplus R_{12} \oplus R_{21} \oplus R_{22}$. Throughout this paper, the notation $a_{i j}$ will denote an arbitrary element of $R_{i j}$ and any element $a \in R$ can be expressed as $a=a_{11}+a_{12}+a_{21}+a_{22}$.

For a prime ring $R$ (i.e., $a R b=\{0\}$ implies $a=0$ or $b=0$ ), we denote the maximal right ring of quotients and the two-sided right ring of quotients of $R$ by $Q_{m r}(R)$ and $Q_{r}(R)$, respectively. Note that $R \subseteq Q_{r}(R) \subseteq Q_{m r}(R)$. The center $C=Z\left(Q_{r}(R)\right)$ of $Q_{r}(R)$ is called the extended centroid of $R$. Note that the extended centroid of any prime ring is a field. The subring $R C$ of $Q_{m r}(R)$ is called the central closure of $R$ which is prime if $R$ is prime. We denote the central closure of $R$ by $T$. More details can be found in Chapter 2 of [2]. 
Lemma 1.5. ([2] Theorem 2.3.4) Let $R$ be a prime ring, and $a, b \in Q_{m r}(R)$. Suppose that $a x b=b x a$ for all $x \in R$. Then $a$ and $b$ are $C$-dependent.

We will make crucial use of the following lemmas.

Lemma 1.6. Let $R$ be a prime ring with a nontrivial idempotent and $i, j, k \in\{1,2\}$. The center of $R$ is denoted by $Z(R)$.

(1) If $a_{i j} x_{j k}=0$ for all $x_{j k} \in R_{j k}$, then $a_{i j}=0$.

(2) If $x_{i j} a_{j k}=0$ for all $x_{i j} \in R_{i j}$, then $a_{j k}=0$.

(3) If $a_{11} x_{12}-x_{12} a_{22}=0$ for all $x_{12} \in R_{12}$, then $a_{11}+a_{22}=z$ for some $z \in Z(R)$.

(4) If $a_{22} x_{21}-x_{21} a_{11}=0$ for all $x_{21} \in R_{21}$, then $a_{11}+a_{22}=z$ for some $z \in Z(R)$.

(5) If $a \in R$ and $\left[a, x_{12}\right]=\left[a, x_{22}\right]=0$ for all $x_{12} \in R_{12}$ and $x_{22} \in R_{22}$, then $a \in Z(R)$.

(6) If $a \in R$ and $\left[a, e_{1}\right]=\left[a, x_{i j}\right]=0$ for all $x_{i j} \in R_{i j}(i \neq j)$, then $a \in Z(R)$.

Proof. (1) and (2) are direct consequences of the primeness of $R$.

(3) For any $x_{11} \in R_{11}$ and $y_{12} \in R_{12}$, we have

$$
a_{11}\left(x_{11} y_{12}\right)=x_{11}\left(y_{12} a_{22}\right)=x_{11} a_{11} y_{12} .
$$

It follows from (1) that

$$
a_{11} x_{11}=x_{11} a_{11} .
$$

Now for any $x_{12} \in R_{12}$ and $y_{22} \in R_{22}$,

$$
\left(x_{12} y_{22}\right) a_{22}=\left(a_{11} x_{12}\right) y_{22}=x_{12} a_{22} y_{22} \text {. }
$$

By (2), we see that

$$
a_{22} y_{22}=y_{22} a_{22} \text {. }
$$

Let $x_{21} \in R_{21}$ and $y_{12} \in R_{12}$ be arbitrary. Applying Identity (1.2), we get

$$
a_{22}\left(x_{21} y_{12}\right)=x_{21}\left(y_{12} a_{22}\right)=x_{21} a_{11} y_{12},
$$

which, by (1), implies that

$$
a_{22} x_{21}=x_{21} a_{11} .
$$

Now, for any $x \in R$, using Identities (1.1), (1.2), and (1.3), we have

$$
\begin{aligned}
\left(a_{11}+a_{22}\right) x & =\left(a_{11}+a_{22}\right)\left(x_{11}+x_{12}+x_{21}+x_{22}\right) \\
& =a_{11} x_{11}+a_{11} x_{12}+a_{22} x_{21}+a_{22} x_{22} \\
& =x_{11} a_{11}+x_{12} a_{22}+x_{21} a_{11}+x_{22} a_{22} \\
& =\left(x_{11}+x_{12}+x_{21}+x_{22}\right)\left(a_{11}+a_{22}\right) \\
& =x\left(a_{11}+a_{22}\right) .
\end{aligned}
$$

This gives us $a_{11}+a_{22}=z \in Z(R)$.

(4) It is similar to (3).

(5) We write $a=\sum_{i, j=1}^{2} a_{i j}, a_{i j} \in R_{i j}$. By $\left[a, x_{22}\right]=0$, we see that $a_{12} x_{22}=$ $x_{22} a_{21}=0$ for all $x_{22} \in R_{22}$. It follows from (1) and (2) that $a_{12}=a_{21}=0$.

Now that $\left[a, x_{12}\right]=0$ implies $a_{11} x_{12}=x_{12} a_{22}$. It follows from (3) that $a_{11}+a_{22} \in$ $Z(R)$, which completes the proof.

(6) It is similar to (5). 
Lemma 1.7. Let $R$ be a prime ring with a nontrivial idempotent and $i, j, k \in\{1,2\}$.

(1) If $a_{i j} \in T_{i j}$ and $a_{i j} x_{j k}=0$ for all $x_{j k} \in R_{j k}$, then $a_{i j}=0$.

(2) If $a_{j k} \in T_{j k}$ and $x_{i j} a_{j k}=0$ for all $x_{i j} \in R_{i j}$, then $a_{j k}=0$.

Proof. We only show (1).

Since $a_{i j} \in T_{i j}$, there exist $a_{i j}^{\prime} \in R_{i j}$ and $c \in C$ such that $a_{i j}=a_{i j}^{\prime} c$. Therefore, $a_{i j} x_{j k}=0$ for all $x_{j k} \in R_{j k}$ is equivalent to $a_{i j}^{\prime} c x e_{k}=0$ for all $x \in R$. It follows that $a_{i j}^{\prime} x e_{k}=0$ since $C$ is a field. By the primeness of $R$, we see that $a_{i j}^{\prime}=0$, and so $a_{i j}=0$.

\section{LIE DERIVABLE MAPPINGS ON PRIME RINGS}

Throughout this section, we always assume that $R$ is a prime ring containing a nontrivial idempotent with center $Z(R)$ and $\delta: R \rightarrow R$ is a Lie derivable mapping.

Lemma 2.1. $\delta(0)=0$.

Proof. It directly follows from $\delta(0)=\delta([0,0])$.

Lemma 2.2. For any $a_{i i} \in R_{i i}$ and $b_{j k} \in R_{j k}(i, j, k \in\{1,2\}$ and $j \neq k)$, there exists $a z_{a_{i i}, b_{j k}} \in Z(R)$ such that

$$
\delta\left(a_{i i}+b_{j k}\right)=\delta\left(a_{i i}\right)+\delta\left(b_{j k}\right)+z_{a_{i i}, b_{j k}} .
$$

Proof. We only prove the case when $i=j=1$ and $k=2$. The rest of the proof goes similarly.

For any $x_{22} \in R_{22}$,

$$
\begin{aligned}
& {\left[\delta\left(a_{11}\right), x_{22}\right]+\left[a_{11}, \delta\left(x_{22}\right)\right]+\left[\delta\left(b_{12}\right), x_{22}\right]+\left[b_{12}, \delta\left(x_{22}\right)\right] } \\
= & \delta\left(\left[a_{11}, x_{22}\right]\right)+\delta\left(\left[b_{12}, x_{22}\right]\right) \\
= & \delta\left(b_{12} x_{22}\right) \\
= & \delta\left(\left[a_{11}+b_{12}, x_{22}\right]\right) \\
= & {\left[\delta\left(a_{11}+b_{12}\right), x_{22}\right]+\left[a_{11}+b_{12}, \delta\left(x_{22}\right)\right] . }
\end{aligned}
$$

This gives us

$$
\left[\delta\left(a_{11}+b_{12}\right)-\delta\left(a_{11}\right)-\delta\left(b_{12}\right), x_{22}\right]=0
$$

For any $x_{12} \in R_{12}$, on one hand we have

$$
\delta\left(\left[a_{11}+b_{12}, x_{12}\right]\right)=\left[\delta\left(a_{11}+b_{12}\right), x_{12}\right]+\left[a_{11}+b_{12}, \delta\left(x_{12}\right)\right] .
$$

On the other hand, we have

$$
\begin{aligned}
& \delta\left(\left[a_{11}+b_{12}, x_{12}\right]\right) \\
= & \delta\left(a_{11} x_{12}\right) \\
= & \delta\left(\left[a_{11}, x_{12}\right]\right)+\delta\left(\left[b_{12}, x_{12}\right]\right) \\
= & {\left[\delta\left(a_{11}\right), x_{12}\right]+\left[a_{11}, \delta\left(x_{12}\right)\right]+\left[\delta\left(b_{12}\right), x_{12}\right]+\left[b_{12}, \delta\left(x_{12}\right)\right] . }
\end{aligned}
$$

So we have that

$$
\left[\delta\left(a_{11}+b_{12}\right)-\delta\left(a_{11}\right)-\delta\left(b_{12}\right), x_{12}\right]=0 .
$$

By Lemma $1.6(5)$, this together with $(2.1)$ implies that $\delta\left(a_{11}+b_{12}\right)-\delta\left(a_{11}\right)-$ $\delta\left(b_{12}\right) \in Z(R)$, as desired. 
Lemma 2.3. (1) $\delta\left(a_{12}+b_{12}\right)=\delta\left(a_{12}\right)+\delta\left(b_{12}\right)$

(2) $\delta\left(a_{21}+b_{21}\right)=\delta\left(a_{21}\right)+\delta\left(b_{21}\right)$.

Proof. We only show (1). One can get (2) in a similar manner.

Using Lemma 2.2, we have

$$
\begin{aligned}
& \delta\left(a_{12}+b_{12}\right) \\
= & \delta\left(\left[e_{1}-a_{12}, e_{1}+b_{12}\right]\right) \\
= & {\left[\delta\left(e_{1}-a_{12}\right), e_{1}+b_{12}\right]+\left[e_{1}-a_{12}, \delta\left(e_{1}+b_{12}\right)\right] } \\
= & {\left[\delta\left(e_{1}\right)+\delta\left(-a_{12}\right), e_{1}+b_{12}\right]+\left[e_{1}-a_{12}, \delta\left(e_{1}\right)+\delta\left(b_{12}\right)\right] } \\
= & \delta\left(\left[e_{1}, e_{1}\right]\right)+\delta\left(\left[-a_{12}, e_{1}\right]\right)+\delta\left(\left[e_{1}, b_{12}\right]\right)+\delta\left(\left[-a_{12}, b_{12}\right]\right) \\
= & \delta\left(a_{12}\right)+\delta\left(b_{12}\right),
\end{aligned}
$$

which completes the proof.

Lemma 2.4. For any $a_{i i}, b_{i i} \in R_{i i}(i \in\{1,2\})$, there exists a $z_{a_{i i}, b_{i i}} \in Z(R)$ such that

$$
\delta\left(a_{i i}+b_{i i}\right)=\delta\left(a_{i i}\right)+\delta\left(b_{i i}\right)+z_{a_{i i}, b_{i i}} .
$$

Proof. Suppose first that $i=1$. For any $x_{22} \in R_{22}$, we have

$$
\begin{aligned}
& {\left[\delta\left(a_{11}\right), x_{22}\right]+\left[a_{11}, \delta\left(x_{22}\right)\right]+\left[\delta\left(b_{11}\right), x_{22}\right]+\left[b_{11}, \delta\left(x_{22}\right)\right] } \\
= & \delta\left(\left[a_{11}, x_{22}\right]\right)+\delta\left(\left[b_{11}, x_{22}\right]\right) \\
= & \delta\left(\left[a_{11}+b_{11}, x_{22}\right]\right) \\
= & {\left[\delta\left(a_{11}+b_{11}\right), x_{22}\right]+\left[a_{11}+b_{11}, \delta\left(x_{22}\right)\right] . }
\end{aligned}
$$

Then we can deduce that

$$
\left[\delta\left(a_{11}+b_{11}\right)-\delta\left(a_{11}\right)-\delta\left(b_{11}\right), x_{22}\right]=0 .
$$

For any $x_{12} \in R_{12}$, we have

$$
\begin{aligned}
& \delta\left(\left[a_{11}+b_{11}, x_{12}\right]\right) \\
= & \delta\left(a_{11} x_{12}+b_{11} x_{12}\right) \\
= & \delta\left(a_{11} x_{12}\right)+\delta\left(b_{11} x_{12}\right) \\
= & \delta\left(\left[a_{11}, x_{12}\right]\right)+\delta\left(\left[b_{11}, x_{12}\right]\right) .
\end{aligned}
$$

Note that in the second equality we are applying Lemma 2.3. Then we can obtain

$$
\left[\delta\left(a_{11}+b_{11}\right)-\delta\left(a_{11}\right)-\delta\left(b_{11}\right), x_{12}\right]=0 .
$$

By Lemma $1.6(5)$, this together with (2.2) implies that $\delta\left(a_{11}+b_{11}\right)-\delta\left(a_{11}\right)-$ $\delta\left(b_{11}\right) \in Z(R)$

In a similar fashion, one can prove the case of $i=2$.

Lemma 2.5. $\delta\left(a_{12}+b_{21}\right)=\delta\left(a_{12}\right)+\delta\left(b_{21}\right)$.

Proof. Write $\delta\left(a_{12}+b_{21}\right)-\delta\left(a_{12}\right)-\delta\left(b_{21}\right)=\sum_{i, j=1}^{2} u_{i j}, u_{i j} \in R_{i j}$. It suffices to show that each $u_{i j}$ is zero. To do this, observe that

$$
\begin{aligned}
& \delta\left(a_{12}+b_{21}\right) \\
= & \delta\left(\left[b_{21}-a_{12}, e_{1}\right]\right) \\
= & {\left[\delta\left(b_{21}-a_{12}\right), e_{1}\right]+\left[b_{21}-a_{12}, \delta\left(e_{1}\right)\right] } \\
= & {\left[\delta\left(b_{21}-a_{12}\right), e_{1}\right]+\delta\left(\left[b_{21}, e_{1}\right]\right)-\left[\delta\left(b_{21}\right), e_{1}\right]+\delta\left(\left[-a_{12}, e_{1}\right]\right)-\left[\delta\left(-a_{12}\right), e_{1}\right] } \\
= & {\left[\delta\left(b_{21}-a_{12}\right)-\delta\left(b_{21}\right)-\delta\left(-a_{12}\right), e_{1}\right]+\delta\left(b_{21}\right)+\delta\left(a_{12}\right) . }
\end{aligned}
$$


This implies

$$
\sum_{i, j=1}^{2} u_{i j}=\left[\delta\left(b_{21}-a_{12}\right)-\delta\left(b_{21}\right)-\delta\left(-a_{12}\right), e_{1}\right] .
$$

Then we can conclude that $u_{11}=u_{22}=0$.

We now consider $\delta\left(\left[a_{12}+b_{21}, x_{12}\right]\right)$ for any $x_{12} \in R_{12}$. On one hand, we have

$$
\delta\left(\left[a_{12}+b_{21}, x_{12}\right]\right)=\left[\delta\left(a_{12}+b_{21}\right), x_{12}\right]+\left[a_{12}+b_{21}, \delta\left(x_{12}\right)\right] .
$$

On the other hand, we also have

$$
\begin{aligned}
& \delta\left(\left[a_{12}+b_{21}, x_{12}\right]\right) \\
= & \delta\left(\left[b_{21}, x_{12}\right]\right) \\
= & \delta\left(\left[a_{12}, x_{12}\right]\right)+\delta\left(\left[b_{21}, x_{12}\right]\right) \\
= & {\left[\delta\left(a_{12}\right), x_{12}\right]+\left[a_{12}, \delta\left(x_{12}\right)\right]+\left[\delta\left(b_{21}\right), x_{12}\right]+\left[b_{21}, \delta\left(x_{12}\right)\right] . }
\end{aligned}
$$

These give us $\left[u_{12}+u_{21}, x_{12}\right]=0$ for all $x_{12} \in R_{12}$, which implies that $u_{21}=0$.

Similarly, by considering $\delta\left(\left[a_{12}+b_{21}, x_{21}\right]\right)$ for arbitrary $x_{21} \in R_{21}$, we can obtain that $u_{12}=0$.

Lemma 2.6. For $a_{11} \in R_{11}$ and $b_{22} \in R_{22}$, there is a $z_{a_{11}, b_{22}} \in Z(R)$ such that

$$
\delta\left(a_{11}+b_{22}\right)=\delta\left(a_{11}\right)+\delta\left(b_{22}\right)+z_{a_{11}, b_{22}} .
$$

Proof. First, we consider $\delta\left(\left[a_{11}+b_{22}, e_{1}\right]\right)$ in two ways:

$$
\delta\left(\left[a_{11}+b_{22}, e_{1}\right]\right)=\left[\delta\left(a_{11}+b_{22}\right), e_{1}\right]+\left[a_{11}+b_{22}, \delta\left(e_{1}\right)\right]
$$

and

$$
\begin{aligned}
& \delta\left(\left[a_{11}+b_{22}, e_{1}\right]\right) \\
= & 0 \\
= & \delta\left(\left[a_{11}, e_{1}\right]\right)+\delta\left(\left[b_{22}, e_{1}\right]\right) \\
= & {\left[\delta\left(a_{11}\right), e_{1}\right]+\left[a_{11}, \delta\left(e_{1}\right)\right]+\left[\delta\left(b_{22}\right), e_{1}\right]+\left[b_{22}, \delta\left(e_{1}\right)\right] . }
\end{aligned}
$$

Consequently,

$$
\left[\delta\left(a_{11}+b_{22}\right)-\delta\left(a_{11}\right)-\delta\left(b_{22}\right), e_{1}\right]=0 .
$$

Next, we consider $\delta\left(\left[a_{11}+b_{22}, x_{12}\right]\right)$ for any $x_{12} \in R_{12}$. By Lemma 2.3 , we have

$$
\begin{aligned}
& \delta\left(\left[a_{11}+b_{22}, x_{12}\right]\right) \\
= & \delta\left(a_{11} x_{12}-x_{12} b_{22}\right) \\
= & \delta\left(a_{11} x_{12}\right)+\delta\left(-x_{12} b_{22}\right) \\
= & \delta\left(\left[a_{11}, x_{12}\right]\right)+\delta\left(\left[b_{22}, x_{12}\right]\right),
\end{aligned}
$$

which, together with $\delta\left(\left[a_{11}+b_{22}, x_{12}\right]\right)=\left[\delta\left(a_{11}+b_{22}\right), x_{12}\right]+\left[a_{11}+b_{22}, \delta\left(x_{12}\right)\right]$, gives us

$$
\left[\delta\left(a_{11}+b_{22}\right)-\delta\left(a_{11}\right)-\delta\left(b_{22}\right), x_{12}\right]=0
$$

By Lemma $1.6(6)$, this along with (2.3) implies that $\delta\left(a_{11}+b_{22}\right)-\delta\left(a_{11}\right)-\delta\left(b_{22}\right) \in$ $Z(R)$.

Lemma 2.7. For any $a_{11} \in R_{11}, b_{21} \in R_{21}$, and $c_{22} \in R_{22}$ there exists a $z_{a_{11}, b_{21}, c_{22}} \in Z(R)$ such that

$$
\delta\left(a_{11}+b_{21}+c_{22}\right)=\delta\left(a_{11}\right)+\delta\left(b_{21}\right)+\delta\left(c_{22}\right)+z_{a_{11}, b_{21}, c_{22}} .
$$


Proof. With similar approach as in the proof of Lemma 2.6, one can get

$$
\left[\delta\left(a_{11}+b_{21}+c_{22}\right)-\delta\left(a_{11}\right)-\delta\left(b_{21}\right)-\delta\left(c_{22}\right), e_{1}\right]=0 .
$$

For arbitrary $x_{21} \in R_{12}$, we compute

$$
\begin{aligned}
& {\left[\delta\left(a_{11}+b_{21}+c_{22}\right), x_{21}\right]+\left[a_{11}+b_{21}+c_{22}, \delta\left(x_{21}\right)\right] } \\
= & \delta\left(\left[a_{11}+b_{21}+c_{22}, x_{21}\right]\right) \\
= & \delta\left(\left[a_{11}+c_{22}, x_{21}\right]\right)+\delta\left(\left[b_{21}, x_{21}\right]\right) \\
= & {\left[\delta\left(a_{11}+c_{22}\right), x_{21}\right]+\left[a_{11}+c_{22}, \delta\left(x_{21}\right)\right]+\left[\delta\left(b_{21}\right), x_{21}\right]+\left[b_{21}, \delta\left(x_{21}\right)\right] } \\
= & {\left[\delta\left(a_{11}\right)+\delta\left(c_{22}\right), x_{21}\right]+\left[a_{11}+c_{22}, \delta\left(x_{21}\right)\right]+\left[\delta\left(b_{21}\right), x_{21}\right]+\left[b_{21}, \delta\left(x_{21}\right)\right] . }
\end{aligned}
$$

Note that in the last equality we are applying Lemma 2.6. Then we can infer that

$$
\left[\delta\left(a_{11}+b_{21}+c_{22}\right)-\delta\left(a_{11}\right)-\delta\left(b_{21}\right)-\delta\left(c_{22}\right), x_{21}\right]=0 .
$$

By Lemma $1.6(6)$, this together with (2.4) implies that $\delta\left(a_{11}+b_{21}+c_{22}\right)-\delta\left(a_{11}\right)-$ $\delta\left(b_{21}\right)-\delta\left(c_{22}\right) \in Z(R)$, which completes the proof.

Lemma 2.8. For any $a_{11} \in R_{11}, b_{12} \in R_{12}, c_{21} \in R_{21}$, and $d_{22} \in R_{22}$, there exists $a z_{a_{11}, b_{12}, c_{21}, d_{22}} \in Z(R)$ such that

$$
\delta\left(a_{11}+b_{12}+c_{21}+d_{22}\right)=\delta\left(a_{11}\right)+\delta\left(b_{12}\right)+\delta\left(c_{21}\right)+\delta\left(d_{22}\right)+z_{a_{11}, b_{12}, c_{21}, d_{22}} .
$$

Proof. From

$$
\begin{aligned}
& \delta\left(\left[a_{11}+b_{12}+c_{21}+d_{22}, e_{1}\right]\right) \\
= & \delta\left(-b_{12}+c_{21}\right) \\
= & \delta\left(-b_{12}\right)+\delta\left(c_{21}\right) \\
= & \delta\left(\left[a_{11}, e_{1}\right]\right)+\delta\left(\left[b_{12}, e_{1}\right]\right)+\delta\left(\left[c_{21}, e_{1}\right]\right)+\delta\left(\left[d_{22}, e_{1}\right]\right),
\end{aligned}
$$

one can easily get

$$
\left[\delta\left(a_{11}+b_{12}+c_{21}+d_{22}\right)-\delta\left(a_{11}\right)-\delta\left(b_{12}\right)-\delta\left(c_{21}\right)-\delta\left(d_{22}\right), e_{1}\right]=0 .
$$

We now consider $\delta\left(\left[a_{11}+b_{12}+c_{21}+d_{22}, x_{12}\right]\right)$ for any $x_{12} \in R_{12}$. Using Lemma 2.7 we obtain

$$
\begin{aligned}
& \delta\left(\left[a_{11}+b_{12}+c_{21}+d_{22}, x_{12}\right]\right) \\
= & \delta\left(\left[a_{11}+c_{21}+d_{22}, x_{12}\right]\right)+\delta\left(\left[b_{12}, x_{12}\right]\right) \\
= & \delta\left(\left[a_{11}, x_{12}\right]\right)+\delta\left(\left[b_{12}, x_{12}\right]\right)+\delta\left(\left[c_{21}, x_{12}\right]\right)+\delta\left(\left[d_{22}, x_{12}\right]\right) .
\end{aligned}
$$

This leads to

$$
\left[\delta\left(a_{11}+b_{12}+c_{21}+d_{22}\right)-\delta\left(a_{11}\right)-\delta\left(b_{12}\right)-\delta\left(c_{21}\right)-\delta\left(d_{22}\right), x_{12}\right]=0 .
$$

Thus, it follows from Lemma $1.6(6)$ that $\delta\left(a_{11}+b_{12}+c_{21}+d_{22}\right)-\delta\left(a_{11}\right)-\delta\left(b_{12}\right)-$ $\delta\left(c_{21}\right)-\delta\left(d_{22}\right) \in Z(R)$. The proof is complete.

Theorem 2.9. Let $R$ be a prime ring containing a nontrivial idempotent. Suppose that mapping $\delta: R \rightarrow R$ satisfies

$$
\delta([a, b])=[\delta(a), b]+[a, \delta(b)]
$$

for all $a, b \in R$. Then there exists $a z_{a, b}$ (depending on $a$ and $b$ ) in $Z(R)$, the center of $R$, such that

$$
\delta(a+b)=\delta(a)+\delta(b)+z_{a, b} .
$$


Proof. For any $a, b \in R$, we write $a=a_{11}+a_{12}+a_{21}+a_{22}$ and $b=b_{11}+b_{12}+b_{21}+b_{22}$. By Lemma 2.8, there exists $z_{1} \in Z(R)$ such that

$$
\begin{aligned}
& \delta\left(\left[\left(a_{11}+b_{11}\right)+\left(a_{12}+b_{12}\right)+\left(a_{21}+b_{21}\right)+\left(a_{22}+b_{22}\right)\right]\right) \\
= & \delta\left(a_{11}+b_{11}\right)+\delta\left(a_{12}+b_{12}\right)+\delta\left(a_{21}+b_{21}\right)+\delta\left(a_{22}+b_{22}\right)+z_{1} .
\end{aligned}
$$

It follows from Lemma 2.4 that there exist $z_{2}, z_{3} \in Z(R)$ such that

$$
\begin{aligned}
& \delta\left(a_{11}+b_{11}\right)=\delta\left(a_{11}\right)+\delta\left(b_{11}\right)+z_{2}, \\
& \delta\left(a_{22}+b_{22}\right)=\delta\left(a_{22}\right)+\delta\left(b_{22}\right)+z_{3} .
\end{aligned}
$$

We also have, by Lemma 2.8 ,

$$
\begin{aligned}
& \delta(a)=\delta\left(a_{11}+a_{12}+a_{21}+a_{22}\right)=\delta\left(a_{11}\right)+\delta\left(a_{12}\right)+\delta\left(a_{21}\right)+\delta\left(a_{22}\right)+z_{4}, \\
& \delta(b)=\delta\left(b_{11}+b_{12}+b_{21}+b_{22}\right)=\delta\left(b_{11}\right)+\delta\left(b_{12}\right)+\delta\left(b_{21}\right)+\delta\left(b_{22}\right)+z_{5},
\end{aligned}
$$

for some $z_{4}, z_{5} \in Z(R)$.

Now, we obtain

$$
\begin{aligned}
& \delta(a+b) \\
= & \delta\left(\left[\left(a_{11}+b_{11}\right)+\left(a_{12}+b_{12}\right)+\left(a_{21}+b_{21}\right)+\left(a_{22}+b_{22}\right)\right]\right) \\
= & \delta\left(a_{11}+b_{11}\right)+\delta\left(a_{12}+b_{12}\right)+\delta\left(a_{21}+b_{21}\right)+\delta\left(a_{22}+b_{22}\right)+z_{1} \\
= & \delta\left(a_{11}\right)+\delta\left(b_{11}\right)+z_{2}+\delta\left(a_{12}\right)+\delta\left(b_{12}\right)+\delta\left(a_{21}\right)+\delta\left(b_{21}\right)+\delta\left(a_{22}\right)+\delta\left(b_{22}\right)+z_{3}+z_{1} \\
= & \delta(a)-z_{4}+z_{2}+\delta(b)-z_{5}+z_{3}+z_{1} \\
= & \delta(a)+\delta(b)+\left(z_{1}+z_{2}+z_{3}-z_{4}-z_{5}\right) .
\end{aligned}
$$

We write $z_{a, b}=z_{1}+z_{2}+z_{3}-z_{4}-z_{5}$ in the above equality, then we arrive at

$$
\delta(a+b)=\delta(a)+\delta(b)+z_{a, b},
$$

as desired.

We now begin to characterize Lie derivable mappings on prime rings as a sum of a derivation and a central mapping vanishing at commutators. We also want to mention that in the rest of this paper we shall follow the line of [9]. Some proofs are just modification of their counterparts in [9]. For the sake of completeness, we give the proofs here.

Lemma 2.10. $\delta\left(e_{1}\right)=\left[e_{1}, s\right]+z$, for some $s \in R$ and $z \in Z(R)$.

Proof. For any $x_{12} \in R_{12}$, we have

$$
\delta\left(x_{12}\right)=\delta\left(\left[e_{1}, x_{12}\right]\right)=\delta\left(e_{1}\right) x_{12}-x_{12} \delta\left(e_{1}\right)+e_{1} \delta\left(x_{12}\right)-\delta\left(x_{12}\right) e_{1} .
$$

Multiplying this equation by $e_{1}$ from the left and by $e_{2}$ from the right, we obtain

$$
e_{1} \delta\left(e_{1}\right) x_{12}-x_{12} \delta\left(e_{1}\right) e_{2}=0 .
$$

Equivalently, $e_{1} \delta\left(e_{1}\right) e_{1} x e_{2}-e_{1} x e_{2} \delta\left(e_{1}\right) e_{2}=0$. It follows from Lemma 1.6 (3) that $e_{1} \delta\left(e_{1}\right) e_{1}+e_{2} \delta\left(e_{1}\right) e_{2}=z \in Z(R)$. Hence, $\delta\left(e_{1}\right)=\left(e_{1} \delta\left(e_{1}\right) e_{2}+e_{2} \delta\left(e_{1}\right) e_{1}\right)+z$. Letting $s=e_{1} \delta\left(e_{1}\right) e_{2}-e_{2} \delta\left(e_{1}\right) e_{1}$, we get $\delta\left(e_{1}\right)=\left[e_{1}, s\right]+z$.

Let $a d_{s}: R \rightarrow R$ denote the inner derivation induced by $s$, that is, $a d_{s}(x)=$ $x s-s x$ for all $x \in R$. We also let $\Delta=\delta-a d_{s}$. Then one can easily verify the following lemma. 
Lemma 2.11. (1) $\Delta$ is a Lie derivable mappings, $i$. e.,

$$
\Delta([a, b])=[\Delta(a), b]+[a, \Delta(b)] \quad \text { for all } a, b \in R .
$$

(2) $\Delta(a+b)=\Delta(a)+\Delta(b)+z_{a, b}$ for some $z_{a, b} \in Z(R)$.

(3) $\Delta$ is additive on $R_{i j}$ for $i \neq j$.

(4) $\Delta\left(e_{1}\right) \in Z(R)$.

Moreover, we also have

Lemma 2.12. $\Delta\left(R_{i j}\right) \subseteq R_{i j}$ for $i \neq j$.

Proof. We only show that $\Delta\left(R_{21}\right) \subseteq R_{21}$. Let $x_{21} \in R_{21}$ and write $\Delta\left(x_{21}\right)=$ $\sum_{i, j=1}^{2} u_{i j}, u_{i j} \in R_{i j}$. Then

$$
\begin{aligned}
\sum_{i, j=1}^{2} u_{i j} & =\Delta\left(x_{21}\right)=\Delta\left(\left[x_{21}, e_{1}\right]\right) \\
& =\left[\Delta\left(x_{21}\right), e_{1}\right]+\left[x_{21}, \Delta\left(e_{1}\right)\right] \\
& =\left[\Delta\left(x_{21}\right), e_{1}\right]=-u_{12}+u_{21} .
\end{aligned}
$$

This implies that $u_{11}=u_{12}=u_{22}=0$, and so $\Delta\left(x_{21}\right) \in R_{21}$.

Lemma 2.13. $\Delta\left(R_{i i}\right) \subseteq T_{i i}+C$.

Proof. Let $a_{11} \in R_{11}$ and $\Delta\left(a_{11}\right)=\sum_{i, j=1}^{2} u_{i j}, u_{i j} \in R_{i j}$. Then

$$
0=\Delta\left(\left[e_{1}, a_{11}\right]\right)=\left[\Delta\left(e_{1}\right), a_{11}\right]+\left[e_{1}, \Delta\left(a_{11}\right)\right]=\left[e_{1}, \Delta\left(a_{11}\right)\right]=u_{12}-u_{21} .
$$

This yields that $u_{12}=u_{21}=0$ and $\Delta\left(a_{11}\right) \in R_{11}+R_{22}$. Similarly, for any $b_{22} \in R_{22}$, $\Delta\left(b_{22}\right) \in R_{11}+R_{22}$.

Suppose that $\Delta\left(a_{11}\right)=u_{11}+u_{22}$ and $\Delta\left(b_{22}\right)=v_{11}+v_{22}$. Then

$$
0=\Delta\left(\left[a_{11}, b_{22}\right]\right)=\left[\Delta\left(a_{11}\right), b_{22}\right]+\left[a_{11}, \Delta\left(b_{22}\right)\right]=\left[u_{22}, b_{22}\right]+\left[a_{11}, v_{11}\right],
$$

which implies that $\left[u_{22}, b_{22}\right]=\left[a_{11}, v_{11}\right]=0$.

Since $\left[u_{22}, b_{22}\right]=0$ for all $b_{22} \in R_{22}$, we have $u_{22} x e_{2}=e_{2} x u_{22}$ for any $x \in R$. Note that both $U_{22}$ and $e_{2}$ are elements of $Q_{m r}(R)$. By Lemma 1.5, $u_{22}=e_{2} c$ for some $c \in C$. Thus

$$
\Delta\left(a_{11}\right)=u_{11}+u_{22}=u_{11}+e_{2} c=u_{11}+\left(1-e_{1}\right) c=\left(u_{11}-e_{1} c\right)+c \in T_{11}+C .
$$

One can get $\Delta\left(R_{22}\right) \subseteq T_{22}+C$ in the same fashion.

Now we can conclude that for each $i=1,2$, there exists a mapping $f_{i}: R_{i i} \rightarrow C$ such that

$$
\Delta\left(a_{i i}\right)-f_{i}\left(a_{i i}\right) \in R_{i i}
$$

Note that $f_{i}$ is well defined. Indeed, suppose that $f_{i}\left(a_{i i}\right)=c_{1} \in C$ and $f_{i}\left(a_{i i}\right)=$ $c_{2} \in C$. Then we have $\Delta\left(a_{i i}\right)-c_{1} \in R_{i i}$ and $\Delta\left(a_{i i}\right)-c_{2} \in R_{i i}$. It follows that $c_{2}-c_{1}=\left(\Delta\left(a_{i i}\right)-c_{1}\right)-\left(\Delta\left(a_{i i}\right)-c_{2}\right) \in T_{i i} \cap C=\{0\}$. So $c_{1}=c_{2}$.

Let's define a mapping $D: R \rightarrow T$ by

$$
D(a)=D\left(\sum_{i, j=1}^{2} a_{i j}\right)=\sum_{i, j=1}^{2} \Delta\left(a_{i j}\right)-f_{1}\left(a_{11}\right)-f_{2}\left(a_{22}\right)
$$

for all $a \in R$. A mapping $\tau: R \rightarrow C$ is then defined by

$$
\tau(a)=\Delta(a)-D(a) \text { for all } a \in R .
$$

We can verify that $D$ has the following properties. 
Lemma 2.14. (1) $D\left(R_{i j}\right) \subseteq R_{i j}(i \neq j)$.

(2) $D\left(R_{i i}\right) \subseteq T_{i i}(i=1,2)$.

(3) $D\left(a_{i j}\right)=\Delta\left(a_{i j}\right)(i \neq j)$.

(4) $D\left(\sum a_{i j}\right)=\sum D\left(a_{i j}\right)$.

(5) $D$ is additive on $R_{i j}(i \neq j)$.

Lemma 2.15. Let $a, b \in R$ and $z_{a, b} \in Z(R)$ such that $\Delta(a+b)=\Delta(a)+\Delta(b)+z_{a, b}$. Then

(1) $\tau(a+b)=\tau(a)+\tau(b)+z_{a, b}$.

(2) $D(a+b)=D(a)+D(b)$.

Proof. (1) It suffices to show that for any $a_{i i}, b_{i i} \in R_{i i}, i=1,2$, there is a $z_{a_{i i},}, b_{i i} \in$ $R_{i i}$ such that $\tau\left(a_{i i}+b_{i i}\right)=\tau\left(a_{i i}\right)+\tau\left(b_{i i}\right)+z_{a_{i i}, b_{i i}}$.

Note that $\Delta$ also satisfies Lemma 2.4. That is, for $a_{i i}, b_{i i} \in R_{i i}$, there is a $z_{a_{i i}, b_{i i}} \in R_{i i}$ such that $\Delta\left(a_{i i}+b_{i i}\right)=\Delta\left(a_{i i}\right)+\Delta\left(b_{i i}\right)+z_{a_{i i}, b_{i i}}$. Now, we have

$$
\begin{aligned}
& \tau\left(a_{i i}+b_{i i}\right)-\tau\left(a_{i i}\right)-\tau\left(b_{i i}\right)-z_{a_{i i}, b_{i i}} \\
= & \Delta\left(a_{i i}+b_{i i}\right)-D\left(a_{i i}+b_{i i}\right)-\Delta\left(a_{i i}\right)+D\left(a_{i i}\right)-\Delta\left(b_{i i}\right)+D\left(b_{i i}\right)-z_{a_{i i}, b_{i i}} \\
= & D\left(a_{i i}\right)+D\left(b_{i i}\right)-D\left(a_{i i}+b_{i i}\right) \in T_{i i} \cap C=\{0\} .
\end{aligned}
$$

(2) It follows directly from (1).

In what follows, we shall assume that $R$ is 2 -torsion free additionally.

Lemma 2.16. $D\left(a_{i j} x a_{i j}\right)=D\left(a_{i j}\right) x a_{i j}+a_{i j} D(x) a_{i j}+a_{i j} x D\left(a_{i j}\right)$ holds true for all $a_{i j} \in R_{i j}(i \neq j)$ and $x \in R$.

Proof. Observe that $2 a_{i j} x a_{i j}=\left[\left[a_{i j}, x\right], a_{i j}\right]$. Then

$$
\begin{aligned}
2 D\left(a_{i j} x a_{i j}\right) & =D\left(2 a_{i j} x a_{i j}\right)=\Delta\left(2 a_{i j} x a_{i j}\right)=\Delta\left(\left[\left[a_{i j}, x\right], a_{i j}\right]\right) \\
& =\left[\left[\Delta\left(a_{i j}\right), x\right], a_{i j}\right]+\left[\left[a_{i j}, \Delta(x)\right], a_{i j}\right]+\left[\left[a_{i j}, x\right], \Delta\left(a_{i j}\right)\right] \\
& =\left[\left[D\left(a_{i j}\right), x\right], a_{i j}\right]+\left[\left[a_{i j}, D(x)+\tau(x)\right], a_{i j}\right]+\left[\left[a_{i j}, x\right], D\left(a_{i j}\right)\right] \\
& =\left[\left[D\left(a_{i j}\right), x\right], a_{i j}\right]+\left[\left[a_{i j}, D(x)\right], a_{i j}\right]+\left[\left[a_{i j}, x\right], D\left(a_{i j}\right)\right] \\
& =2\left[D\left(a_{i j}\right) x a_{i j}+a_{i j} D(x) a_{i j}+a_{i j} x D\left(a_{i j}\right)\right] .
\end{aligned}
$$

It follows that $D\left(a_{i j} x a_{i j}\right)=D\left(a_{i j}\right) x a_{i j}+a_{i j} D(x) a_{i j}+a_{i j} x D\left(a_{i j}\right)$ since $R$ is 2torsion free.

Lemma 2.17. For any $a_{i i} \in R_{i i}$ and $b_{j k} \in R_{j k}(j \neq k)$,

(1) $D\left(a_{i i} b_{j k}\right)=D\left(a_{i i}\right) b_{j k}+a_{i i} D\left(b_{j k}\right)$.

(2) $D\left(b_{j k} a_{i i}\right)=D\left(b_{j k}\right) a_{i i}+b_{j k} D\left(a_{i i}\right)$.

Proof. (1) If $i=j$, let $a_{i i} \in R_{i i}$ and $b_{i k} \in R_{i k}(i \neq k)$. We have

$$
\begin{aligned}
D\left(a_{i i} b_{i k}\right) & =\Delta\left(a_{i i} b_{i k}\right)=\Delta\left[a_{i i}, b_{i k}\right] \\
& =\left[\Delta\left(a_{i i}\right), b_{i k}\right]+\left[a_{i i}, \Delta\left(b_{i k}\right)\right] \\
& =\left[D\left(a_{i i}\right)+\tau\left(a_{i i}\right), b_{i k}\right]+\left[a_{i i}, D\left(b_{i k}\right)\right] \\
& =\left[D\left(a_{i i}\right), b_{i k}\right]+\left[a_{i i}, D\left(b_{i k}\right)\right] \\
& =D\left(a_{i i}\right) b_{i k}+a_{i i} D\left(b_{i k}\right) .
\end{aligned}
$$

If $i \neq j$, let $a_{i i} \in R_{i i}$ and $b_{j i} \in R_{j i}$. We have

$$
D\left(a_{i i} b_{j i}\right)=0=D\left(a_{i i}\right) b_{j i}+a_{i i} D\left(b_{j i}\right) .
$$

In the similar fashion one can get (2). 
Lemma 2.18. For any $a_{i i} \in R_{i i}$ and $b_{j j} \in R_{j j}, D\left(a_{i i} b_{j j}\right)=D\left(a_{i i}\right) b_{j j}+a_{i i} D\left(b_{j j}\right)$.

Proof. If $i=j$, let $a_{i i}, b_{i i} \in R_{i i}$, and $x_{i k} \in R_{i k}(i \neq k)$. Then, using Lemma 2.17 three times, we have

$$
\begin{aligned}
D\left(a_{i i} b_{i i}\right) x_{i k} & =D\left(a_{i i} b_{i i} x_{i k}\right)-a_{i i} b_{i i} D\left(x_{i k}\right) \\
& =D\left(a_{i i}\right) b_{i i} x_{i k}+a_{i i} D\left(b_{i i} x_{i k}\right)-a_{i i} b_{i i} D\left(x_{i k}\right) \\
& =D\left(a_{i i}\right) b_{i i} x_{i k}+a_{i i} D\left(b_{i i}\right) x_{i k}+a_{i i} b_{i i} D\left(x_{i k}\right)-a_{i i} b_{i i} D\left(x_{i k}\right) \\
& =\left[D\left(a_{i i}\right) b_{i i}+a_{i i} D\left(b_{i i}\right)\right] x_{i k} .
\end{aligned}
$$

This leads to $\left[D\left(a_{i i} b_{i i}\right)-D\left(a_{i i}\right) b_{i i}-a_{i i} D\left(b_{i i}\right)\right] x_{i k}=0$ for all $x_{i k} \in R_{i k}$. Applying Lemma 1.7, we have $D\left(a_{i i} b_{i i}\right)=D\left(a_{i i}\right) b_{i i}+a_{i i} D\left(b_{i i}\right)$.

If $i \neq j$, let $a_{i i} \in R_{i i}$ and $b_{j j} \in R_{j j}$. Then

$$
D\left(a_{i i} b_{j j}\right)=0=D\left(a_{i i}\right) b_{j j}+a_{i i} D\left(b_{j j}\right) .
$$

Lemma 2.19. $D$ is an ordinary derivation of $R$ into $T$.

Proof. In view of Lemmas 2.17 and 2.18, we only need to show that $D\left(a_{i j} b_{k l}\right)=$ $D\left(a_{i j}\right) b_{k l}+a_{i j} D\left(b_{k l}\right)$ for any $a_{i j} \in R_{i j}$ and $b_{k l} \in R_{k l}(i \neq j, k \neq l)$.

We assume first that $j=k$, then $l=i$. Let $a_{i j} \in R_{i j}$ and $b_{j i} \in R_{j i}$ be arbitrary. We have

$$
\begin{aligned}
& \tau\left(\left[a_{i j}, b_{j i}\right]\right) \\
= & \Delta\left(\left[a_{i j}, b_{j i}\right]\right)-D\left(\left[a_{i j}, b_{j i}\right]\right) \\
= & {\left[\Delta\left(a_{i j}\right), b_{j i}\right]+\left[a_{i j}, \Delta\left(b_{j i}\right)\right]-D\left(a_{i j} b_{j i}-b_{j i} a_{i j}\right) } \\
= & {\left[D\left(a_{i j}\right), b_{j i}\right]+\left[a_{i j}, D\left(b_{j i}\right)\right]-D\left(a_{i j} b_{j i}\right)+D\left(b_{j i} a_{i j}\right) } \\
= & D\left(a_{i j}\right) b_{j i}-b_{j i} D\left(a_{i j}\right)+a_{i j} D\left(b_{j i}\right)-D\left(b_{j i}\right) a_{i j}-D\left(a_{i j} b_{j i}\right)+D\left(b_{j i} a_{i j}\right),
\end{aligned}
$$

which implies that

$$
\left[D\left(a_{i j}\right) b_{j i}+a_{i j} D\left(b_{j i}\right)-D\left(a_{i j} b_{j i}\right)\right]+\left[D\left(b_{j i} a_{i j}\right)-D\left(b_{j i}\right) a_{i j}-b_{j i} D\left(a_{i j}\right)\right]=c \in C .
$$

If $c=0$, then $\left[D\left(a_{i j}\right) b_{j i}+a_{i j} D\left(b_{j i}\right)-D\left(a_{i j} b_{j i}\right)\right]=-\left[D\left(b_{j i} a_{i j}\right)-D\left(b_{j i}\right) a_{i j}-\right.$ $\left.b_{j i} D\left(a_{i j}\right)\right] \in T_{i i} \cap T_{j j}$ and hence is equal to 0 . If $c \neq 0$, multiplying the above equation on the left by $a_{i j}$, we obtain

$$
a_{i j} D\left(b_{j i} a_{i j}\right)-a_{i j} D\left(b_{j i}\right) a_{i j}-a_{i j} b_{j i} D\left(a_{i j}\right)=a_{i j} c .
$$

It follows from Lemma 2.17 that

$$
D\left(a_{i j} b_{j i} a_{i j}\right)-D\left(a_{i j}\right) b_{j i} a_{i j}-a_{i j} D\left(b_{j i}\right) a_{i j}-a_{i j} b_{j i} D\left(a_{i j}\right)=a_{i j} c .
$$

Applying Lemma 2.16, we see that $a_{i j} c=0$. Since $C$ is a field, it follows that $a_{i j}=0$, a contradiction.

We now assume that $j \neq k$. Then we must have $k=i$ and $l=j$. Let $a_{i j}, b_{i j} \in$ $R_{i j}$. Then

$$
D\left(a_{i j} b_{i j}\right)=0=D\left(a_{i j}\right) b_{i j}+a_{i j} D\left(b_{i j}\right) .
$$

Lemma 2.20. $\tau([a, b])=0$ for any $a, b \in R$. 
Proof. Note that $\tau\left(x_{i j}\right)=0$ for all $x_{i j} \in R_{i j}(i \neq j)$. Thus, by the proof of Lemma 2.19 , it suffices to show that

$$
\tau\left(\left[a_{i i}, b_{i i}\right]\right)=0
$$

for all $a_{i i}, b_{i i} \in R_{i i}, i=1,2$. In fact,

$$
\begin{aligned}
& \tau\left(\left[a_{i i}, b_{i i}\right]\right) \\
= & \Delta\left(\left[a_{i i}, b_{i i}\right]\right)-D\left(\left[a_{i i}, b_{i i}\right]\right) \\
= & {\left[\Delta\left(a_{i i}\right), b_{i i}\right]+\left[a_{i i}, \Delta\left(b_{i i}\right)\right]-D\left(a_{i i} b_{i i}-b_{i i} a_{i i}\right) } \\
= & {\left[D\left(a_{i i}\right)+\tau\left(a_{i i}\right), b_{i i}\right]+\left[a_{i i}, D\left(b_{i i}\right)+\tau\left(b_{i i}\right)\right]-D\left(a_{i i} b_{i i}\right)+D\left(b_{i i} a_{i i}\right) } \\
= & {\left[D\left(a_{i i}\right), b_{i i}\right]+\left[a_{i i}, D\left(b_{i i}\right)\right]-D\left(a_{i i}\right) b_{i i}-a_{i i} D\left(b_{i i}\right)+D\left(b_{i i}\right) a_{i i}+b_{i i} D\left(a_{i i}\right) } \\
= & 0 .
\end{aligned}
$$

Let $d=D+a d_{s}$. Then we can get the following characterization on Lie derivable mappings on prime rings.

Theorem 2.21. Let $R$ be a 2-torsion free prime ring containing a nontrivial idempotent. Suppose that mapping $\delta: R \rightarrow R$ (no additivity is assumed) satisfies

$$
\delta([a, b])=[\delta(a), b]+[a, \delta(b)]
$$

for all $a, b \in R$. Then $\delta$ is of the form $d+\tau$, where $d$ is a derivation of $R$ into its central closure $T$ and $\tau$ is a mapping of $R$ into its extended centroid $C$ with the following properties:

(1) There exists a $z_{a, b}$ (depending on $a$ and b) in $Z(R)$, the center of $R$, such that

(2) $\tau([a, b])=0$ for all $a, b \in R$.

$$
\tau(a+b)=\tau(a)+\tau(b)+z_{a, b} .
$$

In particular, we have the following characterization of Lie derivations on prime rings.

Corollary 2.22. Let $R$ be a 2-torsion free prime ring containing a nontrivial idempotent and $\delta: R \rightarrow R$ be a Lie derivation. Then $\delta$ is of the form $D+\tau$, where $D$ is a derivation of $R$ into its central closure $T$ and $\tau$ is an additive mapping of $R$ into its extended centroid $C$ such that $\tau[a, b]=0$ for all $a, b \in R$.

\section{REFERENCES}

1. Z. Bai, S. Du, J. Hou, Multiplicative Lie isomorphisms between prime rings, Comm. Algebra 36 (2008) 1626-1633.

2. K. I. Beidar, W. S. Martindale III, A. V. Mikhalev, Rings with Generalized Identities, Monographs and Textbooks in Pure and Applied Mathematics, Vol. 196, Marcel Dekker, New York, 1996.

3. M. Brešar, Commuting traces of biadditive mappings, commutativity-preserving mappings and Lie mappings, Trans. Amer. Math. Soc. 335 (1993) 525-546.

4. X. Cheng, W. Jing, Additivity of maps on triangular algebras, Electron. J. Linear Algebra. 17 (2008) 597-615.

5. P. Li, W. Jing, Jordan elementary maps on rings, Linear Algebra Appl. 382 (2004) 237-245.

6. F. Lu, Additivity of Jordan maps on standard operator algebras, Linear Algebra Appl. 357 (2002) 123-131

7. F. Lu and B. Liu, Lie derivable maps on B(X), J. Math. Anal. Appl. 372 (2010), 369-376.

8. W. S. Martindale III, When are multiplicative mappings additive? Proc. Amer. Math. Soc. 21 (1969) 695-698. 
9. W. S. Martindale III, Lie derivations of primitive rings, Michigan Math. J. 11 (1964) 183-187. Department of Mathematics \& Computer Science, Fayetteville State University, FAYETTEVILLE, NC 28301

E-mail address: wjing@uncfsu.edu

Department of Mathematics, Soochow University, Suzhou 215006, P. R. China

E-mail address: fylu@suda.edu.cn 